\title{
Automated assembly of large double-sided microstrip detector modules of the CBM Silicon Tracking System at FAIR
}

\author{
P. Pfistner*, T. Blank, R. Speck, M. Weber, for the CBM collaboration \\ Karlsruhe Institute of Technology, Germany \\ E-mail: patrick.pfistnerakit.edu
}

\begin{abstract}
The detector modules of the Silicon Tracking System of the Compressed Baryonic Matter experiment at FAIR comprise double-sided silicon microstrip sensors with a size of up to $124 \mathrm{~mm}$ x $62 \mathrm{~mm}$. Due to tight material budget constraints, the sensors are connected to the read-out electronics by long flexible microcables. As manual assembly of the modules is time-consuming and difficult, a fully customized in-house bonder machine has been developed which allows for a highly automated detector module assembly. We present the requirements and the setup of the bonder machine together with the achieved alignment accuracy and first assemblies.
\end{abstract}

Topical Workshop on Electronics for Particle Physics TWEPP2019

2-6 September 2019

Santiago de Compostela - Spain

\footnotetext{
*Speaker.
} 


\section{Introduction}

The Silicon Tracking System (STS) of the Compressed Baryonic Matter (CBM) experiment at the future Facility for Antiproton and Ion Research (FAIR) at GSI, Darmstadt, Germany, provides momentum determination and track identification of charged particles created in heavy-ion collisions [1].

The STS consists of eight tracking stations located $0.3 \mathrm{~m}$ to $1 \mathrm{~m}$ downstream of the target with a total area of about $4 \mathrm{~m}^{2}$. The required momentum resolution of $\frac{\Delta p}{p} \approx 2 \%$ imposes tight material budget constraints. Therefore the front-end electronics are located outside the detector acceptance and have to be connected to the sensor by low-mass flexible microcables with a length of up to $50 \mathrm{~cm}$. A total of 896 detector modules will be mounted on lightweight carbon support structures. Including spares, the total production volume will be 1100 modules.

In an attempt to overcome some of the drawbacks (highly manual, time-consuming process) of the primary assembly technology of aluminum-aluminum TAB bonding based on aluminum polyimide microcables [2, 3], a double-layered copper polyimide microcable has been developed at KIT [4]. Together with this copper microcable, which is at the limit of modern fabrication technology, we developed a novel high-density interconnection technology based on solder paste printing on the cable and gold stud bump bonding of the die, both sensor and ASIC [5].

To make full use of the automation capabilities of the solder paste - bump bonding interconnection, a fully customized bonder machine has been designed and constructed in-house. The bonder machine requirements and the realized setup are described in section 2. The working principle of the machine is described in section 3. This is followed by the achieved alignment accuracy and first assembly results presented in section 4 .

Figure 1a shows a single-sided assembly of eight copper microcables on a $6.2 \mathrm{~cm} \times 6.2 \mathrm{~cm}$ sensor while figure $1 \mathrm{~b}$ shows the important dimensions in a side and top view.

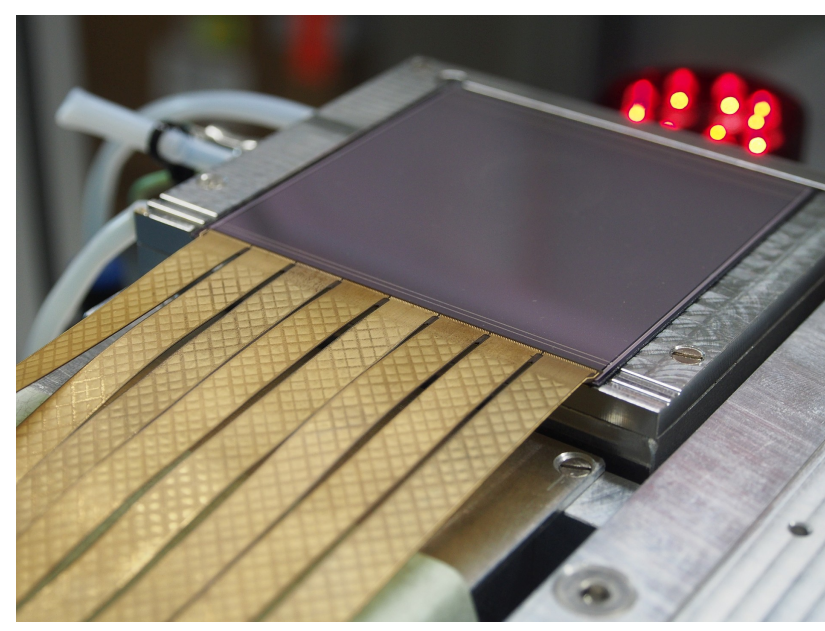

(a) Full single-sided assembly of a $6.2 \mathrm{~cm} \times 6.2 \mathrm{~cm}$ sensor and eight copper microcables.
Side view
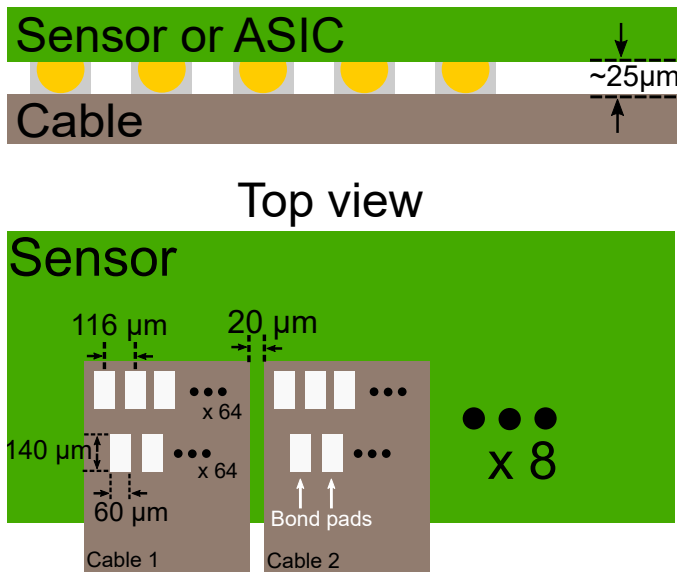

(b) Side and top view sketches showing the important dimensions. 


\section{Automated bonder machine for CBM STS}

A significant number of requirements have to be fulfilled so that the bonder machine is able to reliably interconnect the STS sensors with the microcables.

Alignment accuracy There is only a small gap of $20 \mu \mathrm{m}$ in $\mathrm{x}$-direction between the individual microcables on the sensor which places an upper bound on alignment accuracy of $10 \mu \mathrm{m}$. Furthermore, the microcables are produced in sheets of eight cables and have to be separated with a laser. The tolerance in the lasering of the contours of the microcables currently is $5 \mu \mathrm{m}$. Therefore, an alignment accuracy of $5 \mu \mathrm{m}$ in the $\mathrm{x}$-direction is demanded.

Thermal resistance and thermal stability During the bonding process, temperatures of up to $230^{\circ} \mathrm{C}$ are applied to the bond head and sensor plate. The maximum working temperature of the $\mathrm{z}$-stage is $50^{\circ} \mathrm{C}$ as given by the manufacturer. Therefore, a good thermal insulation between sensor plate and the motorized stages is required. Thermal fluctuations will lead to differences in thermal expansion between the cable and sensor and therefore to inaccuracies in alignment. The thermal stability of the system should be better than $2{ }^{\circ} \mathrm{C}$.

Bond force The machine must be able ro provide a bond force of at least $40 \mathrm{~N}$ [5]. This equates to roughly $30 \mathrm{~g}$ per gold stud. Additionally, the same bond force must be applied over the course of multiple assemblies across several months.

Planarity There are 64 bond pads along the width of the microcable with a pitch of $116 \mu \mathrm{m}$. All channels across the width of the cable have to have a reliable connection. If we allow a maximum height difference across all 64 channels of $10 \mu \mathrm{m}$, the error in planarity between cable and sensor must be smaller than $0.08^{\circ}$.

With the above mentioned requirements in mind, the bonder machine has been designed and constructed. It is shown in figure 2 with its key components labelled:

$1 \& 2$ OWIS ${ }^{\circledR}$ LIMES 150 linear stage for $\mathrm{x}$ and y axes. Repeatability (unidirectional) $<1 \mu \mathrm{m}$.

3 OWIS ${ }^{\circledR}$ HTM 100 z-stage. Repeatability $<10 \mu \mathrm{m}$.

4 OWIS $^{\circledR}$ DMT 100 rotary stage. Repeatability $<0.01^{\circ}$.

5 \& 6 Basler acA1300-200 $\mu$ m camera + Lensation TCST-40-40C lens.

7 Heatable bond head controlled by JUMO Quantrol LC100.

8 Heatable sensor plate controlled by JUMO Quantrol LC100.

\section{Working principle of the bonder machine}

Before the sensor-microcable interconnection can be established, some preparatory steps need to be taken, as described in [4]. In short, the sensor and ASIC [6] are gold stud-bumped. In parallel, both ends of the microcable are printed with fine-grain solder paste, which is subsequently reflowed. Next, ASIC and microcable are connected with a Finetech ${ }^{\circledR}$ FEMTO flip chip machine where the gold-stud bump is pressed into the solder with a bond force of $40 \mathrm{~N}$ at $230{ }^{\circ} \mathrm{C}$ under normal atmosphere. More details of the process can be found in [5]. After this preparation, the 


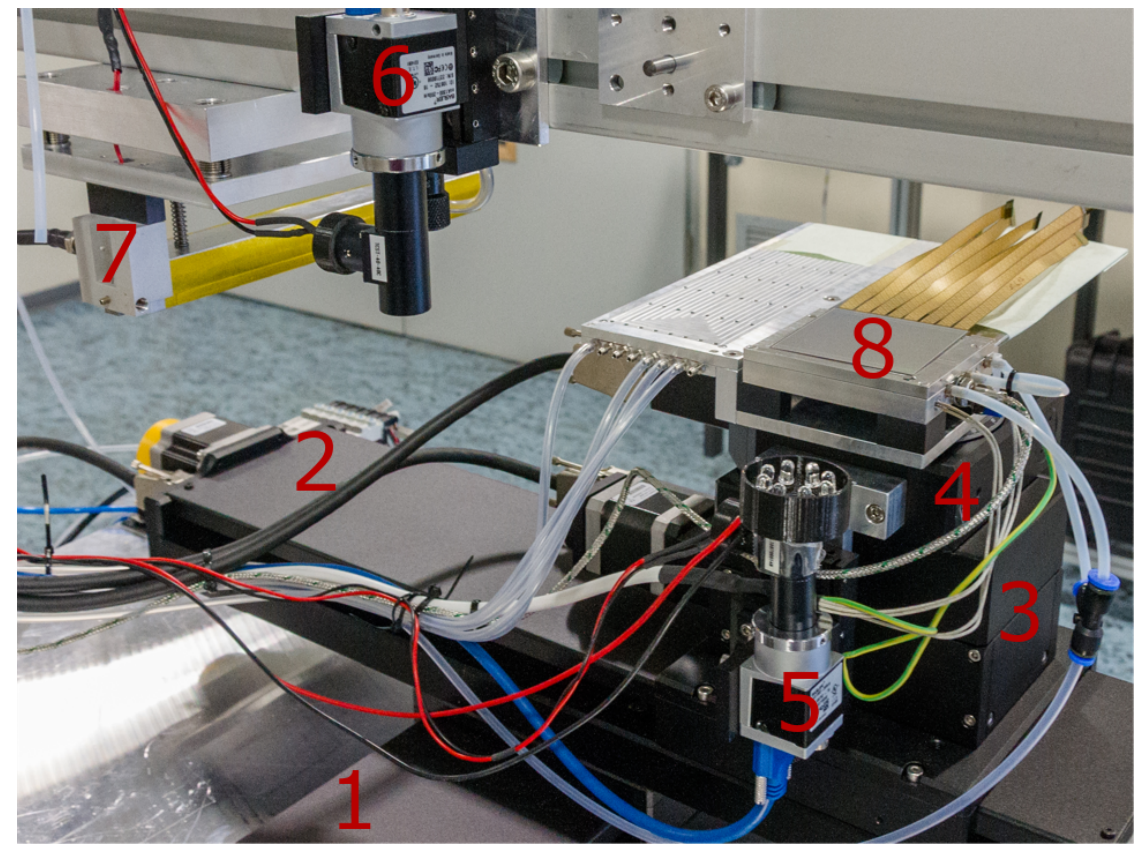

Figure 2: The bonder machine developed at KIT for CBM STS module production. The labelled components are described in the text.

gold-bumped sensor is placed on the sensor plate and eight microcable-ASIC assemblies are put on the vacuum tray. Bond head and sensor plate are ramped up to the respective bonding temperature. The following steps are repeated for all eight cables. First, the microcable is picked up by the bond head. A specially designed vacuum structure keeps the long cable in place. Next, the cable is detected with the bottom camera which moves with the stages. The motor positions and the position of the fiducials in the image are stored.

Then, the stages move so that the top camera is able to detect the sensor via two fiducials in the upper left and upper right sensor corner. The rotational stage rotates the sensor to be parallel to the cable and the fiducials are detected again. If we did not perform a second detection after rotation, we would have to know precisely the distances of the eight assembly positions on the sensor to the rotation axis.

Now, the stages align the first of the eight bonding positions on the sensor with the microcable. The z-stage moves the sensor up into contact where it resides for a typical bonding time of $60 \mathrm{~s}$. Afterwards, the assembly of the next cable can begin.

After one side of the sensor has been fully bonded, underfill glue is dispensed automatically for a given time, pressure and position to strengthen the connection and provide spark protection between the HV sensor and the LV cable. Finally, the full structure is carefully rotated to assemble the opposite side with eight additional cables.

\section{Alignment accuracy}

The final alignment accuracy of the bonding process is determined by various uncertainties. Systematic errors such as the yaw angle and pitch angle of the motors, rotation between bond 
head and motor axes, rotation between individual motors or a tilt between the two cameras can be corrected for by introducing a camera calibration procedure and offsets in the final alignment.

The maximum accuracy of the alignment process is however determined by statistical uncertainties which are mechanical, thermal and optical in nature. They include the repeatability of the linear stages, the camera resolution and the thermal stability of the full system. Five major statistical uncertainties contribute to the bonding process. They have been determined through dedicated tests and were found to be:

- Repeatability of the linear stages $\sigma_{\text {motor }}=2 \mu \mathrm{m}$.

- Accuracy of camera calibration $\sigma_{\text {calib }}=1.5 \mu \mathrm{m}$.

- Accuracy of cable detection $\sigma_{\text {cable }}=2 \mu \mathrm{m}$.

- Accuracy of sensor detection $\sigma_{\text {sens }}=2 \mu \mathrm{m}$.

- Thermal stability $\sigma_{T}=0.5 \mu \mathrm{m}$.

The individual uncertainties are not correlated and add in quadrature to $\sigma_{\text {total }}=3.8 \mu \mathrm{m}$. This value lies well within the specifications of $5 \mu \mathrm{m}$.

\section{Conclusion}

About 1100 large, flexible and therefore delicate detector modules will be built for the CBM STS. To mitigate risks, an alternative microcable and interconnection technology has been developed next to the established aluminum-aluminum TAB bonding technology.

We previously developed a novel die-on-flex high-density interconnection technique based on a fine-pitch double-layered copper microcable. To make full use of its automation capabilities, a fully customized bonder machine has been designed and constructed. Key features include four step motors with sub-micron step resolution, a dual-camera pattern recognition system, a heatable bond head and sensor plate as well as specialized mechanics for the STS detector modules. The final alignment accuracy was found to be less than $5 \mu \mathrm{m}$ which fulfils the requirements for STS. A complete single-sided assembly of a $6.2 \mathrm{~cm} \times 6.2 \mathrm{~cm}$ STS sensor with eight copper microcables has been achieved. To obtain a reliable automated process, the tolerance of the laser separation of the microcables from the sheets will be improved. In addition the bonding temperature will be further reduced to minimize thermal stress on the gold bumps.

\section{References}

[1] J. Heuser et al., Technical Design Report for the CBM STS, Darmstadt, 2013

[2] V.M. Borshchov et al., CBM Progress Report 2013, p. 45, https://repository.gsi.de/record/64893

[3] C. Simons et al., CBM Progress Report 2016, p. 48, https://repository.gsi.de/record/201318

[4] P. Pfistner et al., Novel production method for large double-sided microstrip detectors of the CBM Silicon Tracking System at FAIR, PoS(TWEPP2018)144

[5] P. Pfistner et al., Novel high-density interconnection technology for the CBM Silicon Tracking System, JINST, Vol. 14 Issue 09, doi:10.1088/1748-0221/14/09/p09027

[6] K. Kasinski et al., Characterization of the STS/MUCH-XYTER2, a 128-channel time and amplitude measurement IC for gas and silicon microstrip sensors, Nucl. Instruments Methods Phys. Res. Sect. A, Vol. 30 Issue 9 (2018) pg. 225-235, doi:https://doi.org/10.1016/j.nima.2018.08.076. 